\title{
SEVERE DIASTOLIC \\ SEVERE DIASTOLIC
DYSFUNCTION AFTER ENDOVENTRICULOPLASTY
}

My heart is turn'd to stone;

I strike it and it hurts my hand....

William Shakespeare (Othello)

W e have used the endoventriculoplasty technique (Dor's technique) for a physiologic reconstruction of the aneurysmal left ventricle (LV). ${ }^{1}$ Early reports suggest a better postoperative myocardial performance and, consequently, a lower operative mortality rate than those results obtained with the standard aneurysmectomy. ${ }^{2-4}$ These promising results and the difficulty of heart transplantation inpatients with ischemia encouraged us to perform endoventriculoplasty in patients who were severely ill. After the operation we observed in some patients an acute change of diastolic flow toward a more restric-

From the Division of Thoracic and Cardiovascular Surgery a and the Division of Cardiology, "L. Sacco" Hospital, Via G.B. Grassi, 74, Milan, Italy.

Received for publication March 17, 1994.

Accepted for publication August 26, 1994.

Copyright (C) 1995 by Mosby-Year Book, Inc.

$0022-5223 / 95 \$ 3.00+0 \quad \mathbf{1 2 / 1 / 6 0 2 4 7}$ tive pattern (expression of severe diastolic dysfunction) despite a satisfactory systolic performance.

In the course of our studies we investigated the rate of this acute change of the diastolic properties after endoventriculoplasty along with the clinical consequences and looked for possible explanations for this phenomenon.

\section{Patients and methods}

From 1988 to 199370 patients were referred to us consecutively because of anterior LV aneurysm. At angiography all patients had at least one segment of the LV wall protruding from the normal outline of the ventricular chamber, and this showed either a dyskinetic or akinetic movement. LV performance was evaluated from regional contractility by computerized analysis. ${ }^{3,5}$ The system we used computed a score for each segment so that a normal segment would receive a score of 0 , whereas a fully dyskinetic segment would receive a score of 20 . The global hypokinesis score was the sum of all segmental scores. The aneurysmal score was made up of the anterolateral and apical scores and was assumed as an index of aneurysm extension. The remote myocardial score (outside the aneurysm) was the sum of anterobasal, diaphragmatic, and posterobasal scores.

Fifty-four patients had two-dimensional echocardiography associated with a color Doppler examination before the operation. The transmitral diastolic flow was detected 
by a pulsed Doppler examination at the level of the mitral anulus. Interventricular septum was visualized and considered viable if it showed some systolic thickening, normal or hypokinetic motion, and normal echogenicity. The length of viable interventricular septum was measured from the aortic valvular plane toward the LV apex. Mitral regurgitation was quantified by the left atrium area/ regurgitation jet area ratio and graded from $0+$ to $3+$. Pulmonary arterial hypertension was considered present when the pulmonary arterial systolic pressure was greater than $35 \mathrm{~mm} \mathrm{Hg}$.

All operations were done with cardiopulmonary bypass and aortic crossclamping. Moderate systemic hypothermia $\left(26^{\circ}\right.$ to $28^{\circ} \mathrm{C}$ ), cold antegrade cardioplegia (St. Thomas' Hospital solution), and topical (endocardial and epicardial) cooling were used. Intracardiac repair and myocardial revascularization were done during the same period of aortic occlusion. Left ventriculotomy was begun at the "dimpling" point of the aneurysmal scar and was extended after taking into account the endocardial fibrosis and the presence of muscular tissue. In patients with a large aneurysm a preliminary circumferential suture was used to reduce the mouth of the LV aneurysm. A Dacron fabric or autologous glutaraldehyde-treated pericardial patch was inserted following the junction between scarred and normal endocardium (Fig. 1). The patch was oval with a major width of 3 to $4 \mathrm{~cm}$. The aneurysmal wall was sutured over the patch by means of over-and-over suturing. Biologic glue was applied generously to fill the excluded cavity and to ensure perfect hemostasis.

Myocardial revascularization was done after ventriculotomy and before the patch was applied. All patients but two had myocardial revascularization; 15 patients had single-vessel, 22 had double, 24 had triple, 6 had quadruple, and 1 had quintuple bypass grafting. Revascularization of the left anterior descending artery was accomplished in 45 cases $(64 \%)$.

All survivors underwent two-dimensional echocardiography after operation. The examination was done between 5 and 10 days after the operation. LV volumes, ejection fraction, and apex-base diastolic length (ABL) measurements were taken in 36 patients $(51 \%)$ in whom the echocardiographic windows permitted a reliable visualization of the new LV apex (Fig. 2). In these patients, diastolic volume, systolic volume, and ejection fraction were calculated in the four-chamber view by means of Simpson's model. ${ }^{6}$ The ABL measurement was made from the end-diastolic mitral annular plane to the ventricular patch plane. Normal values for the ABL dimension were derived by the following equation: $Z=66.46+B S A$ 0.41 , where BSA is body surface area. ${ }^{7}$ In two patients with a restrictive diastolic flow pattern, these values were obtained by the transesophageal approach (biplane transducer).

The transmitral flow was detected and the patients were then assigned to groups on the basis of the transmitral flow pattern: group $\mathbf{R}$ comprised patients with a restrictive pattern and group $\mathrm{N}$ comprised patients with a normal or inverted pattern. The restrictive pattern was characterized by a reduced deceleration time, an increased early $\mathrm{E}$ (maximal early diastolic filling velocity), and a reduced late $A$ (peak atrial filling velocity). The inverted pattern was characterized by a reduced early $\mathrm{E}$ and an increased late $\mathrm{A}^{8}$

Intraindividual comparisons of the preoperative and postoperative variables were done with a paired $t$ test. Intergroup comparisons (between groups $R$ and $N$ ) were analyzed by means of a nonpaired $t$ test. Discrete variables were analyzed by means of Fisher's exact test or the $\chi^{2}$ test when appropriate. Probability values of 0.05 or less were considered statistically significant.

\section{Results}

The intraoperative mortality rate was $2.8 \%(2 / 70)$. Both patients who died had failure of LV contractility and could not be weaned from cardiopulmonary bypass despite massive inotropic support and intraaortic balloon counterpulsation. Two other patients died within 30 days of the operation, which raised the global operative mortality rate to $5.7 \%$.

Among the operative survivors, Doppler examination revealed a new restrictive pattern of diastolic flow in six patients $(8.8 \%)$. These six patients had a normal pattern of diastolic flow before operation. The average heart rate of these patients at the time of postoperative Doppler examination was $86 \pm 9$ beats/min. The other patients showed a normal pattern except for six patients who had an inverted pattern after operation. Pertinent clinical data and the outcome of these patients are summarized in Table I. None of these patients had electrocardiographic or enzymatic evidence of postoperative myocardial infarction.

Only two of these six patients (patients 2 and 4) showed a slow but progressive convalescence, and we were able to discharge these patients from the hospital 20 and 25 days, respectively, after the operation. At present, one patient is in New York Heart Association (NYHA) class III with pulmonary hypertension and one is in NYHA class II with moderate mitral regurgitation and normal pulmonary arterial pressure. In both patients the presence of a restrictive diastolic flow was confirmed at follow-up. The other four patients died within 3 months of the operation.

On the other hand, there were no early deaths in the group with normal diastolic flow $(p=0.001)$. Three patients (patients 1, 5, and 6) died of congestive heart failure, and one patient (patient 3 ) died of pneumonia and ventricular fibrillation. In two patients (patients 5 and 6) the hemodynamics were satisfactory in the operating room but deteriorated progressively after the operation. In one of these two patients (patient 6) the intraoperative echocardiogram showed a normal diastolic flow, whereas a 

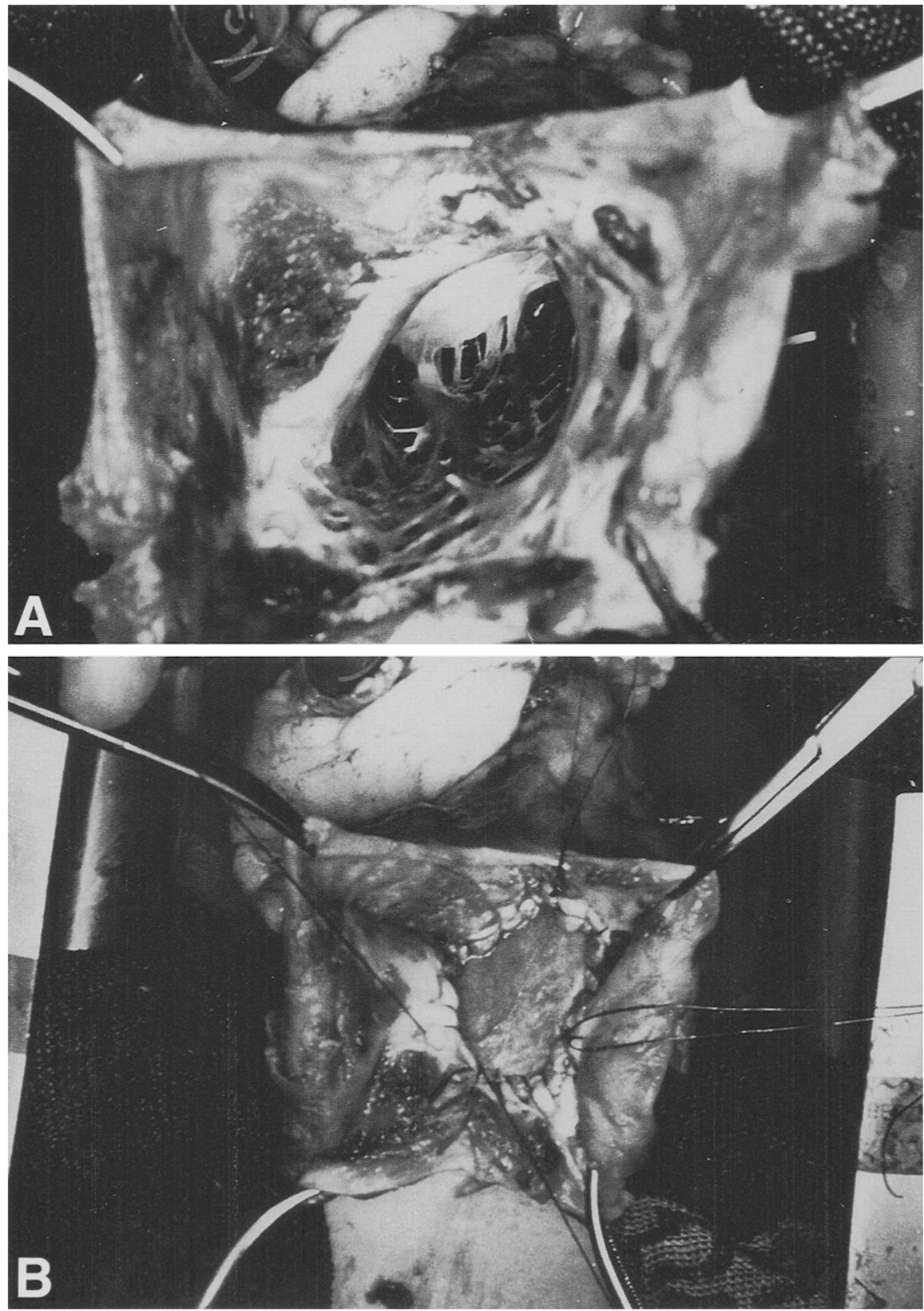

Fig. 1. Intraoperative images of LV aneurysm. A, Operative view of opened LV aneurysm. "Red and white" line is clearly visible, as subvalvular mitral apparatus. Healthy residual cavity is very small. B, Implantation of glutaraldehyde-tanned autologous pericardial patch $(4.5 \mathrm{~cm})$.

restrictive pattern was discovered on the fourth postoperative day. The hemodynamic response of patient 5 to endoventriculoplasty is illustrated in Table II. There was a postoperative rise of pulmonary artery pressure despite an acceptable ejection fraction. The hemodynamic trend worsened when mitral regurgitation recurred and a hypertrophic compensatory response failed to occur.

The preoperative clinical or angiographic differences between the groups are depicted in Table III. The patients with a restrictive flow had clinical and hemodynamic signs of severe impairment that made 


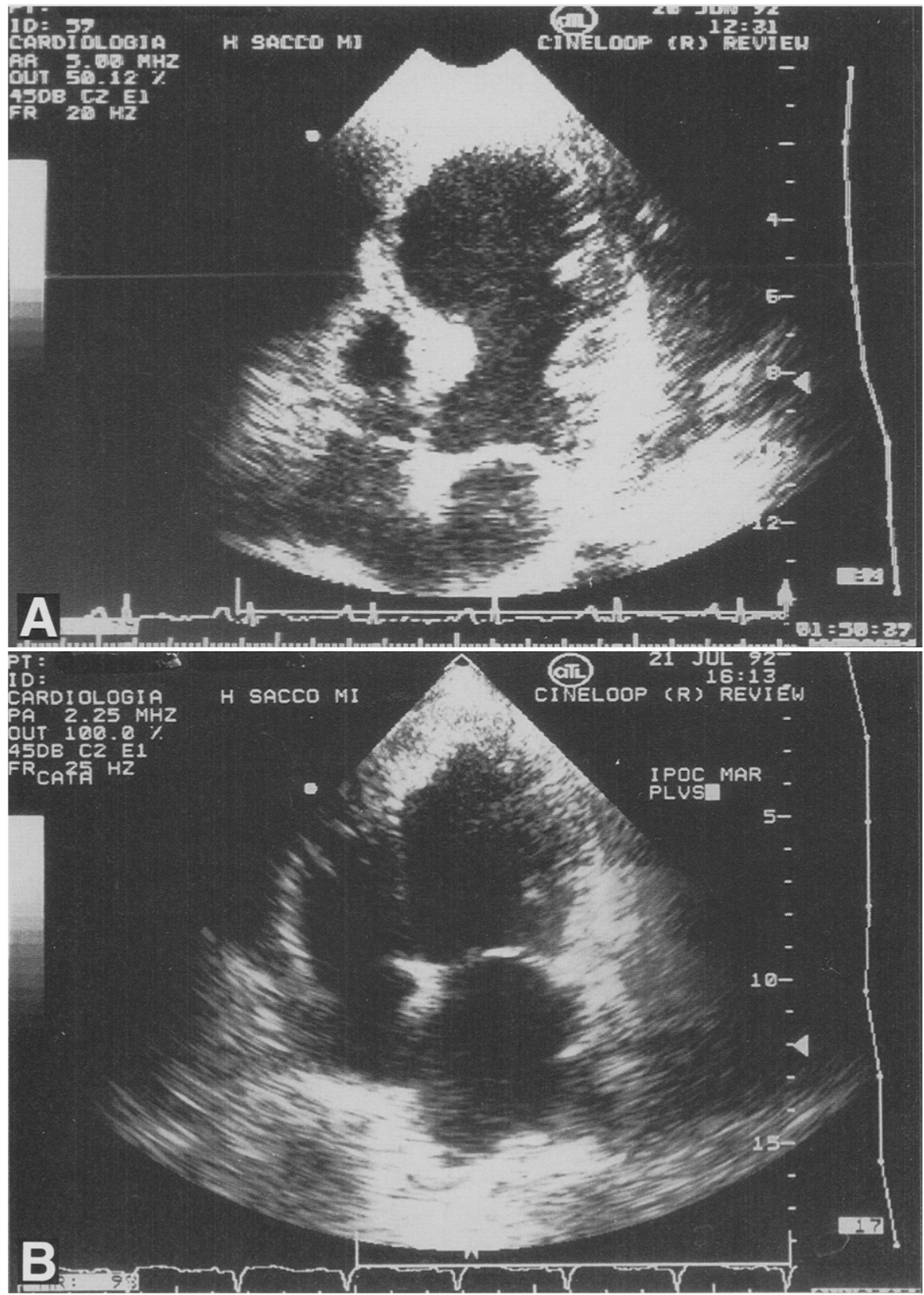

Fig. 2. Echocardiographic images of LV aneurysm. A, Echocardiographic visualization of large anteroseptal aneurysm. Viable septum extends as far as $2 \mathrm{~cm}$ from aortic valve plane. B, Apical four-chamber view showing residual left ventricle after remodeling by endoventriculoplasty. This patient had restrictive pattern of diastolic flow and died 20 days after operation.

it necessary to operate closer to the time of anterior myocardial infarction (Table III). There were no differences between the two groups in regards to remote myocardial contractility and aneurysm extension (Table III). Postoperative echocardiographic examination showed a shortened long-axis measure- ment in the patients with restrictive flow (Table IV), whereas the other LV dimensions did not register any significant difference statistically. Interestingly, the $A B L$ length was less than the predicted value in group $\mathrm{R}(6.5 \pm 0.7$ versus $7.5 \pm 0.26 \mathrm{~cm} ; p<0.05)$, whereas there was no difference in group $\mathrm{N}(7.3 \pm$ 
Table I. Pertinent clinical data for patients with postoperative restrictive pattern of diastolic flow

\begin{tabular}{cccccclc}
\hline Patient & $\begin{array}{c}\text { NYHA } \\
\text { class }\end{array}$ & $\begin{array}{c}\text { Time from } A M I \\
(\mathrm{mo})\end{array}$ & PAH & LABP & MR grade & \multicolumn{1}{c}{ Grafts } & \multicolumn{1}{c}{ Outcome } \\
\hline 1 & III & 2 & N & Y & 2 & IMA-DA, Ao-RCA, Ao-OM & D, 2 mo \\
2 & I-II & 9 & N & N & 2 & IMA-DA & A, 24 mo \\
3 & IV & 1.5 & Y & Y & 1 & None & D, 20 days \\
4 & IV & 2 & Y & Y & 1 & Ao-RCA, Ao-OM & A, 14 mo \\
5 & III & 2.5 & Y & N & 2 & Ao-DA & D, 3 mo \\
6 & III & 1 & Y & N & 2 & Ao-DA & D, 8 days \\
\hline
\end{tabular}

$A M I$, Anterior myocardial infarction; $P A H$, pulmonary arterial hypertension; $L A B P$, intraaortic balloon pulsation (intraoperative); $M R$, mitral regurgitation; $N$, no; $Y$, yes; $I M A$, internal mammary artery; $D A$, descending anterior coronary artery; $A o$, aorta; $R C A$, right coronary artery; $O M$, obtuse marginal branch; $D$, dead; $A$, alive.

Table II. Temporal response to endoventriculoplasty in a patient with postoperative restrictive pattern

\begin{tabular}{lrccc}
\hline & Preop. & 4 days & 1 mo & 2 mo \\
\hline DD (mm) & 70 & 40 & 50 & 21 \\
EDV (ml) & 109 & $*$ & 80 & 92 \\
EF (\%) & 32 & $*$ & 35 & 44 \\
RVP (mm Hg) & 37 & 51 & 60 & 80 \\
MR grade & 3 & 1 & 1 & 2 \\
PWT (mm) & 14 & 13 & 10 & 10 \\
\hline
\end{tabular}

$D D$, Diastolic diameter; $E D V$, end-diastolic volume; $E F$, ejection fraction; $R V P$, right ventricular pressure; $M R$, mitral regurgitation; $P W T$, posterior wall thickness.

*Not measured.

0.6 versus $7.6 \pm 0.2 \mathrm{~cm}$; not significant). Moreover, both groups had a reduced end-diastolic diameter after operation (group $\mathrm{N}$ from $63.4 \pm 10$ to $60.2 \pm$ $6.5 \mathrm{~mm} ; p<0.05$ : group $\mathrm{R}$ from $63.1 \pm 9.5$ to $55.5 \pm$ $4.4 \mathrm{~mm} ; p=0.06$ ) although group $\mathrm{R}$ did not register any statistical significance.

\section{Discussion}

A circular reconstruction for $\mathrm{LV}$ aneurysm has gained wide acceptance for its innovative concept of a restored ventricular geometry. Jatene ${ }^{9}$ performed the circular reconstruction with and without a patch on a transverse plane to restore the natural direction of the myocardial fibers. Dor and associates ${ }^{1}$ improved this technique by putting the patch within the ventricular cavity at the transition zone between scarred and viable myocardium.

This operation seems to overcome the problems related to the standard linear suture because (1) it completely excludes from the ventricular chamber the septal extension of the fibrotic process, (2) it diverts myocardial fibers toward the apex, (3) it decreases the tension on the transitional zone, and (4) it encourages the revascularization of the left anterior descending artery.

This theoretic approach has been confirmed by functional results. Many authors have found a sub- stantial improvement of the ejection-phase indexes (ejection fraction, regional contractility scores). ${ }^{2-4}$ Most important, the wall motion pattern returns to a symmetric mode (symmetric contraction of the opposite walls). This mode of systolic motion was observed in $60 \%$ of the patients who underwent endoventriculoplasty. The other $40 \%$ had a residual deformity of the ventricular chamber with an asymmetric contraction pattern. ${ }^{3}$ We have emphasized the predictive role of a successful geometric reconstruction of the well-preserved proximal interventricular septum. ${ }^{10}$

Clinical data seem to reflect these functional findings. Endoventriculoplasty carries a low operative mortality rate that ranges from $3.3 \%$ to $6.8 \% .^{2-4,9-11}$ Moreover, this rate is similar in every series, which suggests a homogeneous response to the operation. Similar results can be achieved with the linear suture, but there is a wide variation among different series. $^{12}$

On the other hand, the great majority of authors have pointed out the favorable effects on the systolic function whereas postoperative diastolic function has represented the "dark side" of this field. In the past, this has been due to the difficulty in analyzing the factors that influence the relationship between pressure and volume. These factors can be summed up intrinsically as passive muscle stiffness, chamber geometry, relaxation rate, LV suction, and viscoelastic properties of the myocardium and extrinsically as biventricular interaction, erectile effects (the quantity of blood in the coronary arteriovenous bed), heart rate, and pericardial restraint. ${ }^{13}$ The ability to understand these factors has increased considerably in the past decade, and now we can accomplish a semiquantitative evaluation of the diastolic function.

At present, Doppler evaluation of the volumetric filling rate is the most feasible method used to assess diastolic dysfunction in the clinical setting. Gener- 
Volume 109, Number 4

Table III. Preoperative clinical echocardiographic and angiographic data of the patients with restrictive (R) or normal $(N)$ diastolic flow

\begin{tabular}{lccc}
\hline & Group $R(n=6)$ & Group $N(n=62)$ & $p$ Value \\
\hline NYHA class & $2.91 \pm 1.06$ & $1.36 \pm 0.59$ & $<0.001$ \\
BSA (m) & $1.72 \pm 0.14$ & $1.36 \pm 0.59$ & NS \\
Time from MI (mo) & $3.4 \pm 3.0$ & $31.8 \pm 28$ & $<0.05$ \\
EF (\%) & $32.1 \pm 3.2$ & $40.1 \pm 11.5$ & NS \\
LVEDP (mm Hg) & $27.0 \pm 7.2$ & $17.3 \pm 6.8$ & $<0.001$ \\
TS (points) & $57.6 \pm 9.9$ & $45.0 \pm 9.4$ & $<0.05$ \\
AS (points) & $30.0 \pm 4.1$ & $24.9 \pm 7.7$ & NS \\
RS (points) & $27.6 \pm 8.8$ & $19.9 \pm 9.3$ & NS \\
DD (mm) & $63.1 \pm 9.5$ & $63.4 \pm 10$ & NS \\
Viable septum (mm) & $2.0 \pm 1.1$ & $2.8 \pm 1.1$ & NS \\
MR grade & $1.6 \pm 0.5$ & $0.7 \pm 0.6$ & $<0.01$ \\
PAH (\%) & 66.6 & 9.6 & $<0.05$
\end{tabular}

$B S A$, Body surface area; $M I$, myocardial infarction; $E F$, ejection fraction; $L V E D P$, LV end-diastolic pressure; $T S$, total score; $A S$, aneurysmatic score; $R S$, remote score; $D D$, diastolic diameter; $M R$, mitral regurgitation; $P A H$, pulmonary artery hypertension.

Table IV. Postoperative echocardiographic data of the patients with restrictive $(R)$ or normal $(N)$ diastolic flow

\begin{tabular}{lccc} 
& Group $R(n=6)$ & Group $N(n=62)$ & $p$ Value \\
\hline DD $(\mathrm{mm})$ & $55.5 \pm 4.4$ & $60.2 \pm 6.5$ & NS \\
EF $(\%)$ & $39.5 \pm 8.0$ & $39.4 \pm 11.1$ & NS \\
EDV $(\mathrm{ml})$ & $95 \pm 19.2$ & $131 \pm 52$ & NS \\
EDVI & $55.5 \pm 12$ & $75 \pm 33.5$ & NS \\
ABL $(\mathrm{mm})$ & $65 \pm 7$ & $73 \pm 6$ & $<0.01$
\end{tabular}

$D D$, Diastolic diameter; $E F$, ejection fraction; $E D V$, end-diastolic volume; $E D V I$, end-diastolic volume index.

ally, we recognize three different patterns of transmitral diastolic filling: (1) a normal pattern with a predominantly early $\mathrm{E}$ and a consistently late atrial A velocity, (2) an abnormal relaxation pattern with a reversed $\mathrm{E} / \mathrm{A}$ ratio (early $\mathrm{E}<$ late $\mathrm{A}$ velocity), and (3) a restrictive pattern with an increased $\mathrm{E}$ and a reduced A velocity. ${ }^{8}$ In patients with a restrictive pattern, diastolic flow occurs almost exclusively during early diastole, whereas atrial transport becomes negligible. This condition has been reported in patients with significant wall thickening (such as occurs in amyloidosis) or severe systolic dysfunction. ${ }^{8,14}$ Progression from an inverted pattern to a restrictive one leads to a temporary normalization ("pseudonormalization") of the diastolic flow.

We found an abrupt change from a normal preoperative pattern to a restrictive pattern after endoventriculoplasty. This response to the operation involved a small but significant minority of patients $(8 \%)$. This group did not differ from the group with a normal postoperative pattern for preoperative global ejection fraction, extension of the aneurysmal scar, or remote myocardial function. On the contrary, the presence of severe congestive heart failure, pulmonary hypertension, and elevated LV end- diastolic pressure in this group could be attributed to a misleading worse systolic dysfunction or a preoperative diastolic dysfunction. In fact, all these patients had a normal pattern of diastolic flow before operation that could be read as a phase of pseudonormalization.

It is difficult to elucidate the mechanism of this acute worsening of diastolic function. We can exclude the extrinsic factors such as heart rate and pericardial restraint. The abnormal relaxation, an active process, usually presents a different pattern of diastolic flow.

The operation creates a sudden reduction of the ventricular volume, leaving a "shorter" ventricle. Our policy has been to implant the patch at the "red and white" endocardial line. In patients who were operated on shortly after a myocardial infarction (making it impossible to obtain a hypertrophic response) the surgical correction could be overemphasized. In fact, postoperative LV volume is less in the patients with restrictive flow, even if it is at the inferior level of a normal range. Most interestingly, the $A B L$ is significantly shorter than that of patients with postoperative normal diastolic flow and that of the predicted value for a normal population. In fact, 


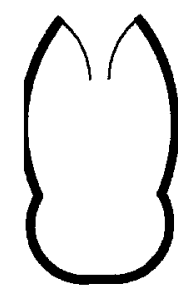

A
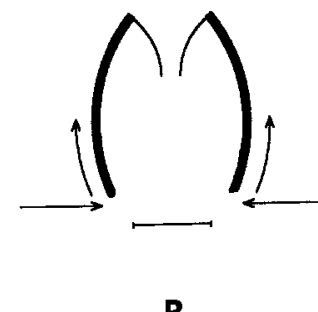

B

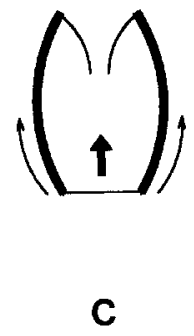

C
Fig. 3. Possible mechanism of diastolic dysfunction after endoventriculoplasty. A, Anteroapical LV aneurysm. B, To implant small patch or to reduce large aneurysmal neck, circumferential stress is developed. This produces longitudinal strain. C, Longitudinal strain pulls patch toward base and interferes with diastolic ventricular suctioning.

it can be enhanced by a longitudinal strain, which occurs in response to compressive forces required to reduce the perimeter of the aneurysmal neck. ${ }^{15}$ These longitudinal forces tend to pull the apical patch toward the base (Fig. 3). Moreover, the presence of longitudinal strain could impair the LV suction. This phenomenon has been found to be an important mechanism in improving ventricular filling when ventricular volume is markedly reduced. ${ }^{16}$ Bleeding in the excluded cavity could hypothetically lead to a compression of the remnant LV. Unfortunately, the postoperative echocardiographic window does not permit a clear visualization of the apical region.

The patients with a postoperative restrictive pattern had a poor prognosis: four patients died within 3 months of the operation. Despite acceptable systolic function, diastolic impairment produced progressive pulmonary hypertension and advanced congestive heart failure. It becomes difficult to control the situation medically when there is a hemodynamic mimicking of constrictive pericarditis (Table II).

In conclusion, caution must be taken when endoventriculoplasty is done in patients in an advanced NYHA class, with pulmonary hypertension, and with elevated LV end-diastolic pressure. Especially in patients very recently affected by myocardial infarction, the operation must be delayed $(>3$ months) to permit the hypertrophic response. When the clinical condition does not permit a delaying of the operation, a more limited correction seems to be a safer option. This could be obtained in two ways: (1) the line of patch implantation should respect the predicted ABL (usually 7.0 to $7.5 \mathrm{~cm}$ ) and (2) the diameter of the patch should be greater $(>5 \mathrm{~cm})$ in high-risk patients. The finding of a restrictive pattern after the operation means strict observation and, when indicated, a semi-urgent heart transplantation can be the only solution.

\section{REFERENCES}

1. Dor V, Saab M, Coste P, Kornaszewska M, Montiglio F. Left ventricular aneurysm: a new surgical approach. Thorac Cardiovasc Surg 1989;37:11-9.

2. Komeda M, David TE, Malik A, Ivanov J, Sun Z. Operative risk and long-term results of operation for left ventricular aneurysm. Ann Thorac Surg 1992;53: 22-9.

3. Salati M, Di Biasi P, Pajè A, Cialfi A, Bozzi G, Santoli C. Functional results of left ventricular reconstruction. Ann Thorac Surg 1993;56:316-22.

4. Mills NL, Everson CT, Hockmuth DR. Technical advances in the treatment of left ventricular aneurysm. Ann Thorac Surg 1993;55:792-800.

5. Bozzi G, Verna E, Skinner JM, Dwyer ML, Castelfranco M. Quantitative regional contraction analysis of cineventriculography reporting, filing, and retrieval functions using a personal computer. Cathet Cardiovasc Diagn 1989;18:50-9.

6. Wyatt HL, Meerbaum S, Heng MK, Gueret P, Corday P. Cross-sectional echocardiography III: analysis of mathematic models for quantifying volume of symmetric and asymmetric left ventricles. Am Heart J 1980;100:821-8.

7. Pearlman JD, Triulzi MO, King ME, Newell J, Weyan AE. Limits of normal left ventricular dimensions in growth and development: analysis of dimensions and variance in the two dimensional echocardiograms of 268 normal healthy subjects. J Am Coll Cardiol 1988;12:1432-44.

8. De Maria AN, Wiswnbaugh TW, Smith MD, Harrison MR, Berk MR. Doppler echocardiographic evaluation of diastolic dysfunction. Circulation 1991; 84(Suppl):I288-95.

9. Jatene AD. Left ventricular aneurysmectomy: resection or reconstruction. J Thorac CARdiovasC SuRg 1985;89:321-31.

10. Salati M, Di Biasi P, Pajè A, Santoli C. Left ventricular geometry after endoventriculoplasty. Eur J Cardiothorac Surg 1993; 7:574-9.

11. Kesler KA, Fiore AC, Naunheim KS, et al. Anterior wall left ventricular aneurysm repair: a comparison of linear versus circular closure. J THORAC CARDIOVASC SURG 1992;103:841-8.

12. Tebbe U, Kreuzer $\mathbf{H}$. Pros and cons of surgery of the left ventricular aneurysm: a review. Thorac Cardiovase Surg 1988;37:3-10.

13. Lewinter MM. Diastolic function and the hypertrophied ventricle. In: Utley JR, ed. Perioperative car- 
diac dysfunction. Baltimore: Williams \& Wilkins, 1985:200-12.

14. Appleton CP, Hatle LK, Popp RL. Demonstration of restrictive ventricular physiology by Doppler echocardiography. J Am Coll Cardiol 1988;11:757-68.

15. Savage EB, Downing SW, Ratcliff MB, et al. Repair of left ventricular aneurysm. J THORAC CARdiovasC SURG 1992;104:752-62.

16. Sabbah HN, Stein PD. Pressure-diameter relations during early diastole in dogs: incompatibility with the concept of passive left ventricular filling. Circ Res 1981;48:357-66.

\section{1-800-55-MOSBY}

This number links you to the full text of articles published in over 25,000 journals, including all Mosby journals. MOSBY Document Express $^{\mathrm{TM}}$, a rapid response information retrieval service, provides quick turnaround, 24-hour availability, and speedy delivery methods. For inquiries and pricing information, call our toll-free, 24-hour order line: 1-800-55-MOSBY; outside the United States: 415-259-5046; fax: 415-259-5019; E-mail: mosbyexp@class.org.

MOSBY Document Express ${ }^{\mathrm{TM}}$ is offered in cooperation with Dynamic Information Corp. 